\title{
Magnetic investigations for studying planetary interiors
}

\author{
Massimo Chiappini and Angelo De Santis \\ Istituto Nazionale di Geofisica, Roma, Italy
}

\begin{abstract}
Most of the magnetic methods used for investigating planetary interiors are based on the reasonable hypothesis that the mechanism for the origin of the field is an Earth-like hydromagnetic dynamo: in this case the planet has an electrically conducting fluid shell within it as in the case of the Earth's core. The present paper describes several techniques of planetary magnetic investigation which give important clues on the internal constitution of planets. Some considerations on the possible mechanisms for maintaining a dynamo and simple concepts with the help of a few non-dimensional numbers are also introduced and discussed. Then some fundamental relationships are given in order to relate the planetary magnetism to other physical parameters, such as angular rotation, core dimensions etc. It finally summarizes some results available for the planets of the Solar System.
\end{abstract}

Key words planetary magnetism - magnetic probing

\section{Introduction}

The characteristic magnetic field $\boldsymbol{B}$ of a planet (when present) is generally a spacetime varying vector field. Some planets of the Solar System, including the Earth, have their own magnetic field of internal origin which can be an important clue for inferring some properties of their internal structure. In an electric-current-free space, if $V$ is the magnetic potential, it is $\boldsymbol{B}=-\nabla V$ and

$$
\nabla^{2} V=0
$$

In cartesian coordinates $x, y, z, \nabla$ is the vector differential operator $\hat{x} \partial / \partial x+\hat{y} \partial / \partial y$ $+\hat{z} \partial / \partial z(\hat{x}, \hat{y}, \hat{z}$ are unit vectors along $x, y, z$ directions, respectively). Equation (1.1) holds in regions close to the planet, where the solar wind-magnetosphere interaction can be neglected, and when the ef- fects of non-conservative fields are small; this latter requirement can be simply satisfied by selecting data only during magnetically quiet time intervals. Hence, in principle, several measurements made within a short period of time and all around the planet allow us to sketch a model of the planetary magnetic morphology.

In fact, in order to realistically analyse the global planetary magnetic field we need to consider two other factors: 1) the larger the distribution of measurements is, the better the knowledge of the real distribution of the potential, around the planet, is achieved; 2) long-lived measurements lead us to estimate possible long-term variation of the field (secular variation).

If one of the above desiderata is not satisfied, data interpretation would be still possible, using the Earth as a comparison, e.g. applying the hydromagnetic geodynamo theory (e.g. Melchior, 1986) to the planet under investigation or considering the dipolar term as the most important energetic contribution ( $>94 \%$ for the Earth) 
to the magnetic field of the planet. In contrast, such a dipolar approximation cannot be applied, for example, to planets like Jupiter, for which the dipolar component is less than $70 \%$ of the whole field.

Some reviews are already available in the literature on magnetic probing of planetary interiors (e.g. Benton, 1979). Together with the best known methods this paper deals with some aspects of their application and with up-to-date results obtained from observations of the planets in the Solar System. Some general considerations, applicable to any planet under some reasonable conditions will be given. Reference can also be made to two previous papers on this topic, specifically on the magnetic investigation of Mercury (Chiappini and De Santis, 1991a,b), this work representing a natural continuation and extension of that study.

\section{Hypotheses on the origin of planetary magnetic fields}

Since this work is intended to be essentially a review of magnetic techniques, in this section only a brief summary of geodynamo theory will be given; for a more detailed description, see, e.g. Roberts and Gubbins (1987) and Soward (1992).

\subsection{Existence of a dynamo}

The magnetohydrodynamic (MHD) approach attempts to estimate a set of fluid motions and fields that obey both the laws of motion and Maxwell's equations. Consider the induction equation (e.g. Stevenson, 1983)

$$
\partial \boldsymbol{B} / \partial t=\nabla \times(\boldsymbol{v} \times \boldsymbol{B})+\eta \nabla^{2} \boldsymbol{B}
$$

where $\eta=(\mu \sigma)^{-1}$ is the magnetic diffusivity, with $\mu$ the magnetic permeability and $\sigma$ the electrical conductivity. It can be seen that the first term on the right-hand side, called the «induction term», describes the possible generation of magnetic field, by interaction with velocity fields $\boldsymbol{v}$ of motions in the conducting fluid core. In the case of a uniform stationary conductor for which $\boldsymbol{v}=0, \partial \boldsymbol{B} / \partial t$ reduces to the second term of eq. (2.1)

$$
\partial \boldsymbol{B} / \partial t=\eta \nabla^{2} \boldsymbol{B}
$$

called the «diffusion term»; it represents the decay of the field, following an inverse exponential time law with a characteristic magnetic diffusion time $\tau$. This characteristic time $\tau\left(\approx R_{c}^{2} / \eta \approx 3000 R_{c}^{2} \times 10^{-6} \mathrm{yr}\right.$ with the planetary core radius $R_{c}$ in $\mathrm{km}$; $\tau \sim 1.2 \times 10^{5} \mathrm{yr}$ for the Earth), is for all planets much less than the age of the Solar System, $4.5 \times 10^{9}$ years.

The possibility of a field being indefinitely maintained is allowed by equation (2.1) because of the induction term. The ratio of this to the diffusion term is the so-called magnetic Reynolds number $R_{m}$ $\left(R_{m}=V L / \eta\right.$, where $V$ and $L$ are a typical velocity and the length scale of the magnetic variations, respectively; in the case of the fluid core of a planet $L \approx R_{c}$ ). The typical state for a working dynamo is reached when the induction is balanced by the diffusion term and $\partial \boldsymbol{B} / \partial t$ has a non-negative average value; this generally happens for $R_{m} \geq 10$ (Stevenson, 1983). Another requirement is that the characteristic time scale of the fluid motions, $L / V$, is much less than the diffusion time $\tau$; because this time is much smaller than the age of the Solar System, it is unlikely that a dynamo can retain information regarding its primordial field. Moreover, such a field must be amplified by electric currents (with associated fields) generated by magnetic fields embedded within the convecting fluid medium. The amplification is necessary to balance the effects of Ohmic dissipation. However, a full description of the problem should require the parallel use of the equation of motion (the so-called Navier-Stokes equation) taking into account also the Coriolis force which is «dynamically important» (Stevenson, 1983). More simply, its contri- 
bution can be considered by introducing the Rossby number, $R_{o}$ ( $=V / 2 \Omega L$, where $\Omega$ is the spin angular velocity of the planet). In principle $R_{o}$ should be less than unity. Another important role is played by the so-called Cowling's theorem (e.g. Roberts and Gubbins, 1987) according to which an axisymmetric magnetic field cannot be self-maintained by the action of a dynamo; this fact seems to be confirmed by planetary magnetic fields which are always tilted with respect to the rotation axis.

A relevant aspect is concerned with the fact that the dynamo process creates both toroidal and poloidal fields (*). The former remain trapped in the planetary conducting core, which is the main source of the whole field, and cannot be measured outside the planet; the latter are the only ones we can measure at the surface of the planet or during a planetary flyby. According to Busse's approach (e.g. 1975, 1983), for the Earth, the balance between the part of the Coriolis force that cannot be balanced by the pressure gradient and Lorentz magnetic forces connected with the core surface (called magnetostrophic balance), allows us to obtain a poloidal field comparable in magnitude to the toroidal field. This fact reduces the energy required to maintain the process.

In addition to the magnetic Reynolds number, Stevenson (1984) related the nature of a planetary dynamo to the so-called energy flux number, which depends on the energy flux, radius of the core, density, angular velocity and the square of the magnetic diffusivity. For dynamo action to be possible, the energy flux number should be greater than 1. For energy-limited and dynamically-determined dynamos it falls in the ranges $1-300$ and $300-100000$, respectively. To give an illustration of the meaning of these ranges, the planet Mercury can be included in the former class, while the Earth may be thought to be in the latter.

(*) Azimuthal and meridional fields are typical simple forms of toroidal and poloidal fields respectively.
However for turbulent dynamos, typical of planets like Jupiter, the energy flux number is greater than $10^{5}$.

\subsection{Energy considerations}

Dynamos can be driven by heat, gravity and rotation, with the processes acting either individually or simultaneously (e.g. Roberts and Gubbins, 1987). For example, according to the "gravitational» dynamo, a possible source for its power supply is located in the core, where separation of a light element, like silicon or sulphur, from a heavy one, essentially iron, implies an energy release. According to this theory, suggested for the Earth by Braginsky (1964), the internal core would have grown because of crystallization of almost pure iron, and the light element exceeding the fluid part of the core would supply the necessary driving force for convection that can be driven by compositional motions because of the presence of alloys with different densities, since (Stevenson, 1983):

$$
E_{\text {grav }} \frac{d m}{d t}>\frac{\triangle T}{T} 4 \pi R_{c}^{2} F_{c}
$$

$\left(E_{\text {grav }}=\right.$ gravitational energy release per unit inner core mass $m ; F_{c}=$ actual core heat flux; $R_{c}=$ outer core radius; $T=$ maximum temperature in the fluid core; $\triangle T=$ temperature difference between $T$ and that of the outer core upper boundary). The mechanisms of other kinds of dynamos are described in detail by Roberts and Gubbins (1987). However, it is reasonable to believe that all mechanisms may coexist at the same time.

\section{Relationships between planetary magnetic field and planetary properties}

\subsection{Magnetic Bode's law}

As a first, extremely empirical, law the so-called magnetic Bode's law has been 
proposed by several authors (e.g. Busse, 1976; Russel, 1979). It derives from the classical Bode's law, that simply relates planetary distances from the Sun with a numerical progression (e.g. Uchupi and Emery, 1993; section 2.2.). In the case of planetary magnetism, the magnetic Bode's law provides a linear relation between the logarithms of magnetic momentum and angular momentum of each planet, both measured in the corresponding Earth units (fig. 1). This is an example of an empirical scaling law mainly based on the geostrophic balance (Busse, 1976). However according to the presently known values, if correct, some planets (e.g. Mars and Venus) seem to deviate from the linear relation, showing a more complex situation than that presented by the over-simple magnetic Bode's law (this could be also explained by the fact that the planets deviating from this empirical law seem not to have any true internal magnetic field). On the other hand, a more recent proposal (Mitzutani et al., 1992),

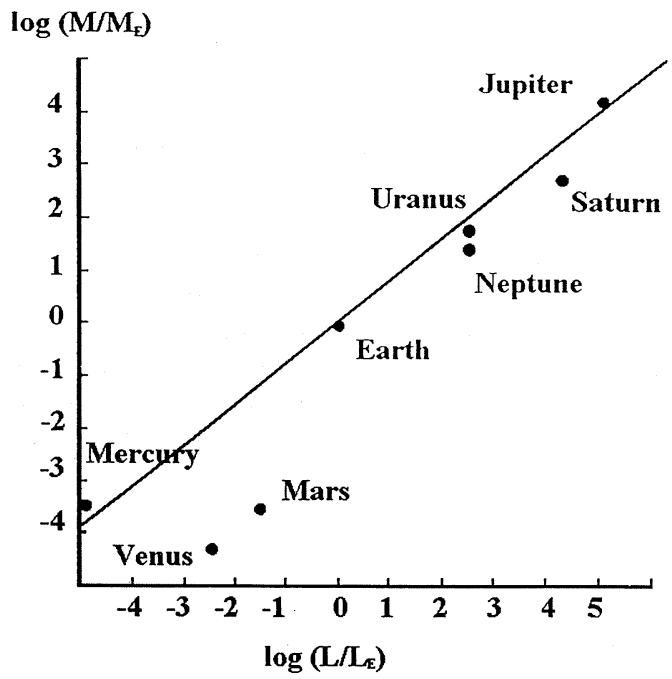

Fig. 1. Magnetic Bode's law for most of the planets of the Solar System (adapted from Rikitake and Honkura, 1985). based on a magnetostrophic balance seems to be more appropriate.

\subsection{Hide's formulation}

In comparison with Bode's law, the following three formulations are more properly relationships between planetary magnetism and the dimensions of the electrically conducting core. It is assumed (closely true) that $\boldsymbol{B}$ is entirely due to electric currents, with density $\boldsymbol{j}$, flowing within the conducting core. Let us define $N(t, r=R)$ as the number of intersections, at time $t$, of magnetic lines of force with a closed surface $\Sigma$, containing the origin and having radius $r=R . N$ is also called the magnetic pole-strength; it is measured in weber or tesla $\cdot \mathrm{m}^{2}$ and can be expressed as (Hide, 1978):

$$
\begin{aligned}
& N(t, r=R)=\oint_{\Sigma}|\boldsymbol{B} \cdot d \boldsymbol{S}|= \\
= & \int_{0}^{2 \pi} \int_{0}^{\pi}\left|B_{r}(r, \theta, \lambda, t)\right| r^{2} \sin \theta d \theta d \lambda
\end{aligned}
$$

where $\theta$ and $\lambda$ are respectively geocentric colatitude and longitude, and $B_{r}$ is the radial component of the magnetic field.

On the reasonable hypothesis that the core is a perfect conductor, we must have (Bondi and Gold, 1950):

$$
\frac{\partial N}{\partial t}=0 \quad \text { at } r=R_{c}
$$

where $R_{c}$ is the planetary core's radius.

For two different epochs and for all surfaces outside the core (e.g. for $r=a>R_{c}$ ) where $\boldsymbol{j}=0$, we may define the quantity:

$$
\triangle N\left(t_{1}, t_{2}, r=a\right)=N\left(t_{2}, r=a\right)-N\left(t_{1}, r=a\right)
$$

Extrapolating $\triangle N$ down to the planetary interior, we will find a particular $r^{*}$ for which $\triangle N=0$. This must hold at the metallic core's equipotential surface (Hide, 
1978). Carrying on this method by small iterations, the value of $r^{*}=R_{c}$ can be computed. First Hide and Malin (1981) and then Voorhies and Benton (1982) applied this computation to the terrestrial case, finding a result that closely confirms the seismic value $(3485 \mathrm{~km})$ for $R_{c}$. Hide's formulation, however, is not applicable to the cases of planetary measurements that are too close in time: eq. (3.3) requires a significant time interval (more than two years) in order to take account of the secular variation. Unfortunately, secular variation has not yet been detected for any other planet than the Earth. It is not yet possible, in fact, to use existing spacecraft data to determine time variations of the intrinsic magnetic field on a time scale of several years (Connerney and Acuña, 1982).

\subsection{Spatial spectrum of the geomagnetic field}

For every contribution associated with $n$, the degree of the harmonic expansion (characterized also by the order $m$ and the harmonic Gauss coefficients $g_{n}^{m}, h_{n}^{m}$ ), the harmonic spatial spectrum of the internal total field $B$ can be defined by the following expression (e.g. Langel and Estes, 1982):

$$
W(n)=(n+1) \sum_{m=0}^{n}\left[\left(g_{n}^{m}\right)^{2}+\left(h_{n}^{m}\right)^{2}\right]
$$

In practice, $W(n)$ is proportional to the mean energy density of $B$ produced by harmonics of a given degree $n$ (Lowes, 1966, 1974). The semi-logarithmic spectrum of $W$ for the Earth is illustrated in fig. 2. Two distinct parts are evident: the first, from harmonic $n=2$ to $n=13$, has the steeper slope and is associated with the core's contribution; the second one (formed by harmonics with $n$ greater than 13 ) has a nearly horizontal slope and is associated with crustal noise. The angular coefficient $\alpha$ of the core part is a function of the core ra-

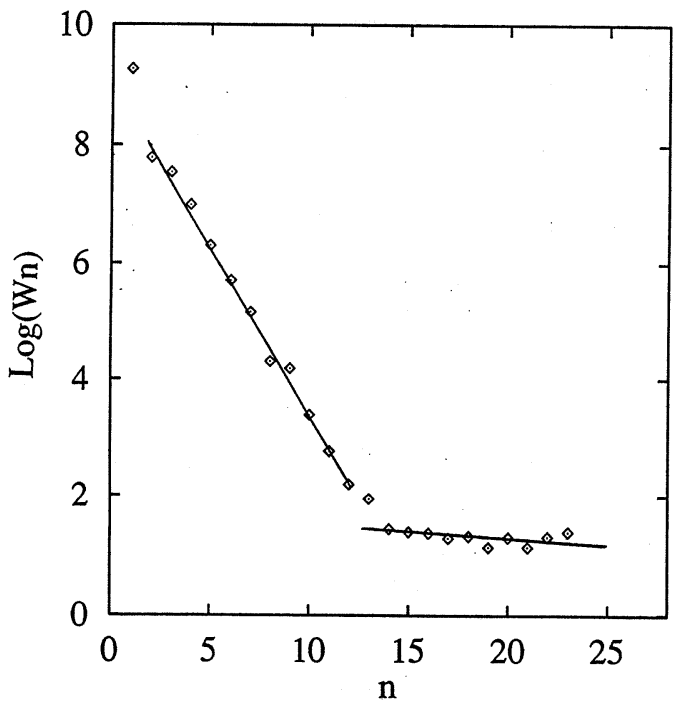

Fig. 2. Spatial spectrum of the Earth's magnetic field: $n$ is the degree of the spherical harmonic expansion of the magnetic field; $W(n)$ is defined by expression (3.4).

dius, through the relation (e.g. Nevanlinna, 1987):

$$
\tan \alpha=2 \log \left(R_{c} / R\right)
$$

where $R$ is the radius of the Earth's surface and $R_{c}$ that of the conducting core.

This relation is derived by assuming a white-noise spectrum at the source layer (e.g. Stevenson, 1983). An alternative view is given by Barraclough (1992) and De Santis and Barraclough (1994) who consider a fractal spectrum at the core-mantle boundary. However, both kinds of analysis require a knowledge of all the harmonic contributions from the core; consequently it appears difficult to apply it to planets other than the Earth.

\subsection{Jacobs' formulation}

Compared with the previous two methods, the following formulation is much sim- 
pler, although it introduces the strongest approximations. Let us consider now only planets whose interiors include a conducting core where a typical hydromagnetic dynamo can take place. It can be supposed that for the value of $B$, measured close to the planetary core's surface, a scaling law holds as follows (Jacobs, 1979a):

$$
B\left(r=R_{c}\right) \propto \sqrt{\varrho_{c}} R_{c} \omega_{c} f\left(R_{m}\right)
$$

where $f\left(R_{m}\right)$ is a function of the magnetic Reynolds number $R_{m}, \varrho_{c}$ is the density of the core and $\omega_{c}$ is the planetary core rotational angular velocity. At the planet's surface $(r=R)$ relation (3.6) becomes (Jacobs, 1979b):

$B(r=R) \propto \sqrt{\varrho_{c}} R_{c} \omega_{c} f\left(R_{m}\right)\left(\frac{R_{c}}{R}\right)^{3}$

Assuming that the proportionality constant and $f\left(R_{m}\right)$ in eq. (3.7) are the same for all planets, the core radius of a planet which is different from the Earth can be deduced, by using our planet as a comparison. Relation (3.7), calculated first for the Earth and then for another planet, gives the ratio:

$$
\frac{B_{E}}{B_{P}}=\left(\frac{\varrho_{E}}{\varrho_{P}}\right)^{1 / 2} \frac{R_{c}^{(E)}}{R_{c}^{(P)}} \cdot \frac{\omega_{E}}{\omega_{P}}\left(\frac{R_{c}^{(E)}}{R_{E}} \cdot \frac{R_{P}}{R_{c}^{(P)}}\right)^{3}
$$

where indices $E$ and $P$ indicate terms associated with the Earth and with the planet under investigation, respectively. The values of planetary fields are computed in the equatorial plane and, to a close approximation, for $\omega_{E} / \omega_{P}$ it is possible to use the ratio between planetary rotational velocities instead of those typical of the cores.

Jacobs (1979a,b), applying the above method to some planets of the Solar System, estimated the radii of their conducting cores $R_{c}^{(P)}$ by means of

$$
R_{c}^{(P)}=R_{c}^{(E)}\left[\frac{B_{P}}{B_{E}}\left(\frac{\varrho_{E}}{\varrho_{P}}\right)^{1 / 2}\left(\frac{R_{P}}{R_{E}}\right)^{3} \frac{\omega_{E}}{\omega_{P}}\right]^{1 / 4}
$$

The values so estimated are shown in table I; some of them (Saturn and Jupiter) are comparable with their respective surface radius. Dolginov (1992) proposed a similar formula (but with an exponent $1 / 6$ instead of $1 / 4$ in (3.9)), calling it the «numerical law» of the precession dynamo model.

\subsection{Adjustment of Jacobs' formulation}

The value of $R_{c}$, as determined by Jacobs' formulation, is to be considered a lower estimation of the real value (Russel, 1987) since this method makes the assumption that the core is a uniformly conducting sphere with radius $R_{c}^{(P)}$ in which electric currents, responsible for the magnetic field observed at the surface, are generated. Chiappini and De Santis (1991a) supposed the existence of a conducting spherical shell, where turbulent motions generate the planetary magnetic field. The magnetic field $\boldsymbol{B}$ generated by a volume current $\boldsymbol{J}$ is given by the expression:

$$
\begin{aligned}
& \boldsymbol{B}(\boldsymbol{x})= \frac{\mu_{0}}{4 \pi} \int \frac{\boldsymbol{J}\left(\boldsymbol{x}^{\prime}\right) \times\left(\boldsymbol{x}-\boldsymbol{x}^{\prime}\right)}{\left|\boldsymbol{x}-\boldsymbol{x}^{\prime}\right|^{3}} d^{3} x^{\prime}= \\
&=\frac{\mu_{0}}{4 \pi} \int \boldsymbol{K}\left(\boldsymbol{x}, \boldsymbol{x}^{\prime}\right) d^{3} x^{\prime}
\end{aligned}
$$

Table I. Values (in $\mathrm{km}$ ) of the metallic core (electrically conductive fluid shell in Jupiter and Saturn) radius as derived from Jacobs' formulation for some planets with supposed magnetic field.

\begin{tabular}{lr}
\hline \hline Mercury & 1100 \\
Venus & 2300 \\
Mars & 200 \\
Jupiter & 74000 \\
Saturn & 60000 \\
\hline
\end{tabular}


where $\boldsymbol{x}$ and $\boldsymbol{x}^{\prime}$ indicate respectively the coordinates of the point where the potential is determined, and the coordinates of the infinitesimal volume $d^{3} x^{\prime}$ of the region where the current density $\boldsymbol{J}\left(\boldsymbol{x}^{\prime}\right)$ flows. Let us consider a spherical shell defined between $r=R_{1}$ and $r=R_{2}$ (with $R_{2}>R_{1}$ ) with volume equal to that of the sphere with radius determined by Jacobs' formulation. Let us introduce the (strong) approximation that $\boldsymbol{K}=$ constant. That means that the spherical shell has a magnetic field equal to that of the sphere previously determined (with volume $\left.4 / 3 \pi\left[R_{c}{ }^{(M)}\right]^{3}\right)$. Hence, the value of $R_{c}$ as computed by Jacobs, can be considered as an intermediate value between $R_{1}$ and $R_{2}$, characterizing the inner and outer core radii, respectively.

\section{Observations}

\subsection{Mercury}

In order to have a picture of the possible problems one can meet in studying planetary interiors by means of magnetic investigations, for the first case of Mercury we will give rather more details than for the other planets.

During its $1974-1975$ voyage, Mariner 10 showed the existence of a weak magnetic field that, extrapolated to Mercury's surface, seemed to have an intensity of several hundreds of nT (Ness et al., 1975). Moreover a magnetosphere, analogous to the Earth's, was observed.

To explain the observed Hermean magnetic moment (around $3 \times 10^{19} \mathrm{~A} \cdot \mathrm{m}^{2} ;$ e.g. Russel, 1987) neither thermal residual magnetization nor induced magnetization by solar wind are sufficient.

Extrapolating Mercury's surface field down to the surface of its core, we find a value of about $3 \mu \mathrm{T}$, i.e. $1 \%$ of the presumed poloidal field in the terrestrial core. On the other hand, since Mercury is slowly rotating (rotational period of 58.8 terrestrial days), a quantitative magnetostrophic balance would lead to a value for the toroidal field of twice that of the Earth, i.e. $2 \cdot 10^{4} \mu \mathrm{T}$ (Gubbins, 1977). This appears unlikely, as for keeping a stable situation, extremely high azimuthal fluid core flow velocities would be required, but this is usually connected with rapid rotations, which is not the case for Mercury. So one is led to conclude that magnetic forces in Mercury must be rather small. The most important equilibrium is thus between the Coriolis and the pressure forces which, for the Earth, is called geostrophic equilibrium. This is one of the basic concepts of Busse's dynamo (Busse, 1975, 1976, 1983).

In order to estimate the energy developed by this mechanism, Gubbins (1977) applied Braginsky's results to the case of Mercury, and supposed a model involving $10 \%$ of lighter elements (density around 6 $\mathrm{g} / \mathrm{cm}^{3}$ ) and $90 \%$ of iron (density about $9 \mathrm{~g}$ / $\mathrm{cm}^{3}$ ). In the same way we can suppose the initial existence of a uniform core with 1600 $\mathrm{km}$ radius and containing a dense mixture with a density of $8.7 \mathrm{~g} / \mathrm{cm}^{3}$. Subsequently a differentiation would have occurred probably releasing roughly $10^{28} \mathrm{~J}$. To be in the range of typical dynamo conditions, we usually suppose a metallic, partially fluid core with a $F e$ alloy constituent which raises the melting point.

For a $\mathrm{Fe}-\mathrm{S}$ model, pressure and temperature conditions within the planet constrain the fluid core thickness to be around 100.p $\mathrm{km}$, where $p=$ initial percentage of $S$ mass. For a reasonable value of $p=3.5 \%$ this thickness would be $350 \mathrm{~km}$. In fig. 3 a possible internal Hermean configuration is shown.

Among the magnetic methods previously described, only Jacobs' formulation and its adjustment appear to be applicable to Mercury. Let us recall that Jacobs' formulation can be used only for planets other than the Earth, since our planet acts as a comparison.

Applying the appropriate adjustment to Jacobs' formulation (Chiappini and De Santis, 1991a) and considering a $350 \mathrm{~km}$ thick spherical shell, a region with $1250 \mathrm{~km}$ internal and $1600 \mathrm{~km}$ external radius is 
Table II. Physical and magnetic properties of planets (adapted from Stevenson, 1983, updating values to more recent results).

\begin{tabular}{lccccc}
\hline \hline Planet & $\begin{array}{c}\text { Mass } \\
(\text { Earth }=1)\end{array}$ & $\begin{array}{c}\text { Period } \\
(\text { Earth }=1)\end{array}$ & $\begin{array}{c}\text { Equat. radius } \\
(\mathrm{km})\end{array}$ & $\begin{array}{c}\text { Equat. } \\
\text { surface field } \\
(\mu \mathrm{T})\end{array}$ & $\begin{array}{c}\text { Dipole tilt } \\
(\mathrm{deg})\end{array}$ \\
\hline Mercury & 0.055 & 58.78 & 2440 & $\sim 2 \times 10^{-1}$ & $\sim 10$ \\
Venus & 0.815 & 243.71 & 6050 & $\left(\leq 2 \times 10^{-3}\right)$ & $(?)$ \\
Earth & 1 & 1 & 6380 & 30 & 11 \\
Mars & 0.107 & 1.028 & 3390 & $\left(\leq 10^{-2}\right)$ & $(\sim 12)$ \\
Jupiter & 318 & 0.411 & 71490 & $\sim 10^{3}$ & $\sim 10$ \\
Saturn & 95 & 0.428 & 60270 & 20 & $\leq 1$ \\
Uranus & 14.95 & 0.748 & 25560 & $\sim 20$ & $\sim 60$ \\
Neptune & 17.1 & 0.802 & 24760 & $\sim 10$ & $\sim 50$ \\
Pluto & $\sim 2 \times 10^{-3}$ & 6.405 & $\sim 1150$ & $(?)$ & $(?)$ \\
\hline
\end{tabular}

found. The agreement with results deduced using different assumptions (Siegfred and Solomon, 1974) is quite good. Figure 3 shows a possible scheme for the planetary interior. Taking account of the fact that $R_{M}=2440 \mathrm{~km}($ e.g. Betty and Chaikin, 1990 ; table II) the volume of the core (external fluid + internal solid) is $28 \%$ of the

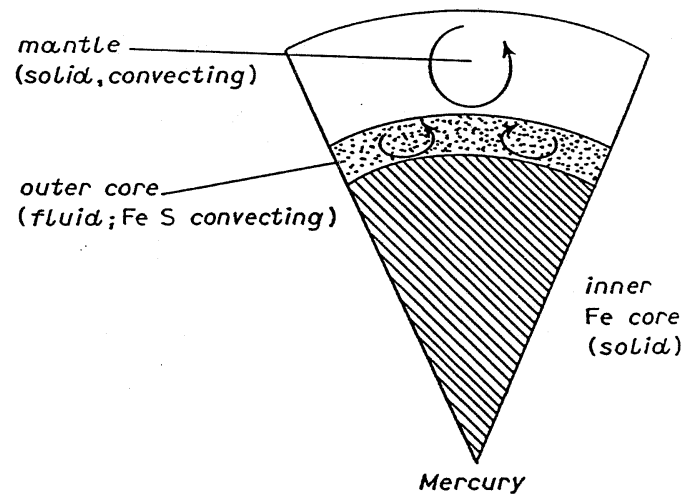

Fig. 3. Internal structure of Mercury (adapted from Stevenson, 1983). total volume, which is a much larger fraction than that observed for the Earth $(16 \%)$.

We can therefore expect a quite different situation compared with the terrestrial case because the surface of Mercury's core is closer to the external surface. Using as a comparison the harmonic spectrum (3.4) for the Earth, a core contribution with $n$ up to almost $20\left(n_{\max }=2 \pi R_{M} /\left[R_{M}-R_{c}^{(M)}\right]\right)$ is expected, while in the case of the Earth $n_{\max }$ is around 13. Furthermore we cannot exclude a richer multipolar contribution with respect to that deduced only from the dipolar hypothesis.

\subsection{Venus}

Among the several satellite missions to the planet Venus, Veneras 9 and 10, in October 1975 , led to the observation of a magnetic tail extending to long distances from the planet. Unfortunately this is not a significant indication of intrinsic planetary magnetism. Dubinin et al. (1978) showed, after laboratory experiments, that a long magnetic tail may be seen also behind an 
unmagnetized body within a magnetic wind.

Later, in December 1978, the Pioneer Venus Orbiter (PVO) observed in the ionosphere of Venus a small magnetic field whose intensity was less than a few nT (Russel et al., 1979a,b). In 1982, Knudsen et al. correlated the steady radial magnetic structures found in the center of the nightside of the planet with ionospheric density depletions called ionospheric holes. Luhmann and Russel (1983) examined the dependence of the magnetic field in these holes on the direction of the interplanetary field; the result of this analysis has led to the understanding that these fields are essentially induced by the solar-wind interaction. This means that the magnetic field direction in the tail depends on the direction of the azimuthal component of the interplanetary magnetic field.

\subsection{Mars}

The planet Mars, whose radius is 3390 $\mathrm{km}$, has been probed by Mariner 4, (July 1965) Mars 2, 3 and 5 (November 1971, December 1971 and February 1974, respectively) and Phobos 2 (February 1989). A region behind the planet was detected, that Dolginov et al. (1976) identified as Martian magnetotail. However, no apparent dayside entry into the magnetosphere was observed, rather a little solar-wind deflection. The topology of the magnetic field of the Martian magnetosphere is different from that of Venus.

Dolginov (1992) estimated the order of magnitude of the Martian magnetic moment to be $2.5 \times 10^{19} \mathrm{~A} \mathrm{~m}^{2}$ with a tilt of $12^{\circ} \pm 3^{\circ}$. If we consider that Mars has a rotational period close to that of the Earth and a core size comparable to that of Mercury (see above), we should hypothesize a field strength between that of the Earth and that of Mercury. Under these considerations, rescaling from the terrestrial dynamo mechanism by using Busse's (1976) theory, we obtain an order of magnitude of the magnetic moment for Mars $10^{3}$ times greater than the observed moment.

Solar-wind interactions with Mars are dominated by induction effects (Schwingenschuh et al., 1992) and the present magnetic field can be considered as the product of a magnetized rock mantle (Yeroshenko, 1992). Consequently, it seems quite probable that even if a dynamo was once present, it has now definitely stopped (Dolginov, 1992).

\subsection{Jupiter}

Among the planets of the Solar System, Jupiter is the largest (equatorial radius, $\left.R_{J}=71500 \mathrm{~km}\right)$ and the most rapidly rotating (period $T=9 \mathrm{~h} 55 \mathrm{~min} 30 \mathrm{~s}$ ). It is accepted that it has a strong magnetic field. Already in 1955, Burke and Franklin detected radio emissions in association with accelerated particles in Jupiter's magnetosphere at a frequency of $22.2 \mathrm{MHz}$, giving evidence of the presence of a planetary magnetic field. Jovian dynamo is the best studied after that of the Earth. The magnetopause of this big planet has been probed by four spacecraft (Pioneer 10 in December 1973, Pioneer 11 in December 1974, Voyager 1 and 2 in March and August 1979, respectively). Within the Jovian magnetosphere, it is thought that the physical processes are somewhat different from those occurring in the terrestrial magnetosphere because of the presence of the Galilean satellite, Io, which is a strong source of plasma, deep in the Jovian magnetosphere, and because of Jupiter's strong magnetic field and of the rapid planetary rotation.

Especially for this latter property, its magnetosphere differs greatly from the Earth's. For modelling purposes, Jupiter's magnetosphere can be divided into three regions: 1) the outer part which is dominated by magnetopause currents on the dayside and the tail currents at night; 2) the middle part which is mainly characterized by the equatorial azimuthal current sheet, and 3) the inner magnetosphere which is 
dominated by the internal planetary field. Connerney et al. (1982) modelled the current sheet with an annular disk of finite thickness, and used it to improve the determination of Jupiter's internal field. Connerney and Acuña (1982) have used the new internal field models to conclude that the Jovimagnetic secular variation must have a time scale of centuries.

From all such evidences, the planetary interior of Jupiter is very different from the interiors of the terrestrial planets, consisting mainly of liquid hydrogen and helium. Because of the high pressure in the planet, its deepest interior is expected to be liquid metallic hydrogen already at $0.71-0.75 R_{j}$; it is within this metallic liquid that Jupiter's dynamo is supposed to act (Russel, 1987). In a near future the Galileo spacecraft, which was launched in 1983, will approach the Jovian magnetosphere; this will be of great value for the knowledge of the dynamics of this planetary magnetosphere and of the intrinsic internal magnetism (e.g. Kivelson et al., 1992).

\subsection{Saturn}

Pioneer 11 in September 1979 and later Voyager 2, in August 1981, reached Saturn, crossing the planet's ring system. The magnetic moment was found to be less than $4.7 \times 10^{25} \mathrm{~A} \mathrm{~m}^{3}$, much less than had been expected from radio emissions (Brown, 1975). The peak of the spectrum of Saturnian emissions is about one eighth that of the Jovian peak, and the measurements of Saturnian field indicate that $B \approx 20000 \mathrm{nT}$ at the equator. Moreover, since the field of Saturn is expected to arise from an internal source in the planet deeper than that from which the field of Jupiter arises (Elphic and Russel, 1978), one would expect the Saturnian field to be more dipolar. If we suppose an outer radius of the magnetic field generation shell of $23000 \mathrm{~km}$ which is consistent with the magnitude of the dipole moment, and repeat the analysis of Elphic and Russel, the expected moment ratio (dipole: quadrupole:octupole) is 1.00:0.12:0.05 compared to the terrestrial ratio of 1.00: 0.14:0.10. Thus Saturn's field should look much more like a dipole than do the terrestrial or Jovian fields.

\subsection{Uranus}

Very little was known about the planet Uranus between the time of its discovery in 1781 by William Herschel, and the Voyager 2 close encounter on 24 January 1986 . Precise observations indicate that the planetary rotation axis is tilted $98^{\circ}$ with respect to its orbit around the Sun. Uranus's orbital period is 84 years at a mean orbital distance of nearly $20 \mathrm{AU}$. The probe's entry into the Uranian magnetosphere revealed the existence of an internal field whose most unexpected property was the large $\left(60^{\circ}\right)$ angle between the dipole and the rotation axes (Ness et al., 1986), in contrast with what happens in the case of the other «magnetic» planets which exhibit a close alignment between their magnetic dipole and rotation axes $\left(\leq 10^{\circ}\right)$, thought to be a natural consequence of the (not satisfactorily understood) dynamo processes. A preliminary simple model (offset, tilted dipole or OTD model), obtained from the magnetic field observations, provides a useful and easily visualized representation of the complex planetary magnetic field of Uranus in terms of a tilted dipole with an offset of 0.33 planet radii $\left(R_{U}=25560 \mathrm{~km}\right.$, table II). However, the problem regarding the large difference between the rotation and magnetic axes is still open. If this tilt is related to that between the rotation and orbit axes then the Uranian interior (where the dynamo is located) should «know» where the Sun is, since the Sun defines the orbit. Ness et al. (1986) proposed that the planet is in a state of magnetic field reversal to explain both the magnetic tilt and the offset dipole; an alternative explanation could be that the source of the field (the dynamo) is closer to the surface than in other planets.

The discovery of a planetary magnetic 
field has been important for another reason connected with the shape of the planet. It has yielded an exact determination (in addition to that from radio emission detection) of the rotational period $(17.24 \mathrm{~h})$, from periodic fluctuations of the magnetic field intensity. This period furnishes meaningful information about the planetary interior, since the rotation produces the equatorial bulge whose size depends on the internal planetary mass distribution. On Uranus, the equatorial radius is $2.4 \%$ greater than the polar one. Measurements carried out by Voyager 2 better fit a twolayer model, which has an internal core and a dense atmosphere composed of $\mathrm{NH}_{3}$, $\mathrm{CH}_{4}$ and $\mathrm{H}_{2} \mathrm{O}$, extending from the core to the surface.

Another peculiar effect of the large tilt of the magnetic axis (pole-on configuration) is that magnetic reconnection between the interplanetary and planetary fields occurs at the time scale of the planetary rotation period, so rapidly that the magnetosphere is a quasi-static MHD entity (McNutt, 1991).

\subsection{Neptune and Pluto}

Very little is known about such remote planets. Neptune was discovered in 1846 by Galle and D'Arrest on the basis of gravitational considerations and after the theoreticians J.C. Adams and Le Verrier already predicted, a few years before, the existence of the planet. Recently, Voyager 2 during its 1989 encounter with Neptune showed a large magnetic field tilt of $47^{\circ}$, with a corresponding surface dipole value of $13.3 \mu \mathrm{T}$, but with a large offset of around half the planetary radius (Ness et al., 1989). Neptune's magnetosphere looks like Uranus's, with a pole-on configuration, so similar situations are expected; also a high-order structure of the magnetic field has been found showing a complicated field as well (Krimigis, 1992). Connerney et al. (1992) describe a spherical harmonic model of the
Neptunian magnetic field up to a maximum degree of 8 .

Regarding Pluto, only some speculative studies have been made. It was discovered only in 1930 by C.W. Tombaugh because of its small size (radius $26 \%$ of the Earth's). Because of its relatively light mass $(0.2 \%$ of the Earth's) it is thought to be composed mostly by ice plus a little rock (Russel, 1987). It is guessed that probably it does not have any active dynamo even if a remanent magnetism could be found.

\section{Final remarks}

After some general considerations on the dynamo process and the ways it can be maintained, we have introduced some magnetic techniques from which it is possible, in principle, to investigate planetary interiors.

Only a brief mention has been made here of planetary magnetospheres which can give further information about the interplanetary and planetary magnetic field coupling and, consequently, can help to better understand some mechanisms responsible for relevant phenomena like e.g. auroras, bow shocks, radio emissions, magnetotail variations, and are in some way related to the properties of the planetary atmospheres and their internal structures (see for example McNutt, 1991).

For surface inaccessible planets it is impossible at present to detect the planetary surfaces seismically in order to investigate their internal constitution. As a reliable alternative, the magnetic investigation has been described with a review of some techniques and their possible application to the planets of the Solar System. In the case of a few planets, where the core has already solidified, the most reasonable explanation for the existence of planetary magnetism is crustal magnetization. However, almost all the techniques assume the existence of a metallic turbulent fluid core inside the planet. Nevertheless other characteristics are also relatively important, for example 
Table III. Interpretation for planetary magnetic fields (adapted from Stevenson, 1983, updating values to more recent results).

\begin{tabular}{lcl}
\hline \hline \multicolumn{1}{c}{ Planet } & $\begin{array}{c}\text { Observed } \\
\text { surface field } \\
(\mu \mathrm{T})\end{array}$ & \multicolumn{1}{c}{ Interpretation } \\
\hline Mercury & $\sim 2 \times 10^{-1}$ & Thin shell dynamo \\
Venus & $\leq 2 \times 10^{-3}$ & Stable, fluid core; no dynamo \\
Earth & 30 & Dynamo maintained by inner-core freezing \\
Mars & $\leq 10^{-2}$ & Stable, fluid $(S$-rich) core; no dynamo \\
Jupiter & $5 \times 10^{2}$ & Dynamo maintained by thermal convection \\
Saturn & 20 & Dynamo maintained by thermal convection \\
Uranus, Neptune & $\sim 10$ & Dynamo in $F e$ core only \\
\hline
\end{tabular}

the planetary core velocity, dimensions, observed surface magnetic field and so on. A general overview is given in table III, revised from Stevenson (1983) with the most recent findings.

Unfortunately, at the moment, dynamo theory is not so advanced as to be able to give either a relationship between the magnetic field intensity and the rotation rate, or a threshold above which a magnetic field can be generated and maintained. Some recent works (e.g. Zhang and Busse, 1990) shed some further light on the subject. Moreover, progress on the understanding of dynamic processes which are associated with the problem of the magnetohydrodynamic geodynamo, is quite slow. Some inconsistencies related to significant differences between the magnetic and the rotational axes (as on Uranus and Neptune) are still a field of investigation, but no easy solutions appear to have yet been found.

In conclusion, we can affirm that the study of planetary magnetic fields can give important advances to the knowledge of the planetary interiors. Moreover, comparative studies can help by giving a better idea about the main global characteristics that drive and govern the origin, formation and maintainance of planetary internal structures and magnetic fields.

\section{Acknowledgements}

The authors would like to thank D.R. Barraclough, J. Brestensky', G.P. Gregori and A. Meloni for their kind suggestions and helpful comments.

\section{REFERENCES}

BARRAClOUGH, D.R. (1992): The geomagnetic scalar potential at the core-mantle boundary. Spatial power spectra and fractal dimension, Geomagn. Group Rep., 92/20, 39.

BEnTON, E.R. (1979): Magnetic probing of planetary interiors, Phys. Earth Planet. Interiors, 20, 111118.

BetTy, J.K. and A. ChaIkin (1990): The New Solar System (Cambridge Univ. Press, New York), 3rd edition.

Bond, H. and T. GoLD (1950): On the generation of magnetism by fluid motion, Mon. Not. R. Astr. Soc., 110, 607-611.

BRAGINSKY, S. I. (1964): Kinematic models of the Earth's core, Geomagn. Aeron., 4, 572-583.

Brown, L.W. (1975): Saturn radio emission near 1 MHz, Astrophys. J., 198, L89-L92.

Burke, B.F. and K.L. FrankLIN (1955): Observations of variable radio source associated with the planet Jupiter, J. Geophys. Res., 60, 213-217. 
Busse, F.H. (1975): A model of the geodynamo, Geophys. J. R. Astron. Soc., 42, 437-459.

BuSSE, F.H. (1976): Generation of planetary magnetism by convection, Phys. Earth Planet. Inter., 12,350 .

Busse, F.H. (1983): Recent developments in the dynamo theory of planetary magnetism, Ann. Rev. Earth Planet. Sci., 11, 241-268.

Chiappini, M. and A. DE SANTis (1991a): Adjustment of Jacobs' formulation to the case of Mercury, Il Nuovo Cimento, 14C (2), 197-201.

Chiappini, M. and A. DE SANTIS (1991b): Study of the Mercury magnetic field for the investigation of its internal structure, in Proceedings of the 2nd Conf. of Geomagnetism and Aeronomy, 267-282, (in Italian).

Connerney, J.E.P. and M.H. Acuña (1982): Jovimagnetic secular variation, Nature, 297, 313-315.

ConNerney, J.E.P., M.H. ACUÑA and N.F. NESS (1982): Voyager 1 assessments of Jupiter's planetary magnetic field, J. Geophys. Res., 87 (A5), 3623.

Connerney, J.E.P., M.H. ACUÑA and N.F. NESS (1992): The magnetic field of Neptune, $A d v$. Space Res., 12 (8), 239-248.

De Santis, A. and D.R. Barraclough (1994): Fractal topography of the geomagnetic scalar potential of the core-mantle boundary, in abstract SE13/ NP1.3, EGS Conference, Grenoble.

Dolginov, Sh.Sh., Ye.G. Yeroshenko and L.N. ZHUZGOV (1976): The magnetic field of Mars according to the data from the Mars 3 and Mars 5, J. Geophys. Res., 81, 3353-3362.

Dolginov, SH.SH. (1992): The magnetic field and the magnetosphere of the planet Mars, Adv. Space Res., 12 (8), 187-211.

Dubinin, E.M., I.M. Podgorny, Ya.N. Potanin and S. I. SHKol'NiKova (1978): Determining the magnetic moment of Venus by magnetic measurements in the tail, Kosmich Issled., 16, 870-876.

ElPHIC, R.C. and C.T. RuSSEL (1978): On the apparent source depth of planetary magnetic fields, Geophys. Res. Lett., 5, 211-214.

GubBINS, D. (1977): Speculation on the origin of the magnetic field of Mercury, Icarus, 30, 186-191.

Hide, R. (1978): How to locate the electrically conducting fluid core of a planet from external magnetic observations, Nature, 271, 640-641.

Hide, R. and S.R.C. MALIN (1981): On the determination of the size of the Earth's core from observations of the geomagnetic secular variation, Proc. R. Soc. London, A374, 15-33.

JACOBS, J.A. (1979a): Planetary magnetic fields, Geophys. Res. Lett., 6, 213-214.

JACOBS, J.A. (1979b): Planetary magnetic fields - errata, Geophys. Res. Lett., 6, 632.

Kivelson, M.G., K.K. Khurana, J.D. Means, C.T. RuSSEL and R.C. SNAKE (1992): The Galileo magnetic field investigation, Space Sci. Rev., 60, 357383.

Knudsen, W.C., P.M. Banks and K.C. Miller (1982): A new concept of plasma motion and plan- etary magnetic field for Venus, Geophys. Res. Lett., 9, 765-768.

Krimigis, S.M. (1992): Particles and fields measurements at Neptune with Voyager 2, Adv. Space Res., 12 (11), 55-70.

LANGEL, R.A. and R.H. Estes (1982): A geomagnetic field spectrum, Geophys. Res. Lett., 9, 250253.

LowES, F.J. (1966): Mean-square values on sphere of spherical harmonic vector fields, J. Geophys. Res., 71, 2179.

LOWES, F.J. (1974): Spatial power spectrum of the main geomagnetic field, and extrapolation to the core, Geophys. J. R. Astron. Soc., 36, 717-730.

LuHMANN, J.G. and C.T. RuSsEL (1983): Magnetic fields in the ionospheric holes of Venus: evidence for an intrinsic field?, Geophys. Res. Lett., 10, 409412.

MCNUTT, R.L.JR. (1991): The magnetospheres of the outer planets, Rev. Geophys., 4, 985-997.

Melchior, P. (1986): The physics of the Earth's core (Pergamon Press, Oxford), 256.

Mitzutani, H., T. Yamamoto and A. Fujimura (1992): A new scaling law of the planetary magnetic field, Adv. Space Res., 12 (8), 265-279.

Ness, N.F., N.F. Behannon, R.P. LePPing and Y.C. WANG (1975): The magnetic field of Mercury: Part 1, J. Geophys. Res., 80, 2708-2714.

Ness, N.S., M.H. ACuña, F.W. Behannon, L.F. Burlaga, J.E.P. ConNerney, R.P. LePPing and F.M. NeUbauer (1986): Magnetic fields at Uræanus, Science, 223, 85-89.

Ness, N.S., M.H. ACUÑA, L.F. Burlaga, J.E.P. ConNERnEY, R.P. LEPPING and F.M. NEUBAuER (1989): Magnetic fields at Neptune, Science, 246, 1473-1478.

NevanlinNA, H. (1987): Notes on global mean-square values of the geomagnetic field and its secular variation, J. Geomagn. Geoelectr., 34, 165-174.

Rikitake, T. and Y. Honkura (1985): Solid Earth Geomagnetism (Terra Sc. Publ. Co., Tokyo).

RoBerTs, P.H. and D. GubBINS (1987): Origin of the main field: kinematics, in Geomagnetism, edited by J.A. JACOBS, 185-249.

RuSSEl, C.T. (1979): Planetary magnetism, Rev. Geophys. Space Phys., 17 (2), 295-301.

RuSSEL, C.T. (1987): Planetary magnetism, in Geomagnetism, edited by J.A. JACOBS, vol. 2, 457 523.

Russel, C.T., R.C. ElPhic and J.A. Slavin (1979a): Initial Pioneer Venus magnetic field results: dayside observations, Science, 203, 745-748.

Russel, C.T., R.C. ElPhiC and J.A. Slavin (1979b): Initial Pioneer Venus magnetic field results: nightside observations, Science, 205, 114-116.

SchwingenschuH, K., W. Riedler, T.L. Zhang, H. Lichtenegger, H. Rosenbauer, S. Livi, G. GevaI, K. Gringauz, M. Verigin, Ye. Yeroshenko, D. Möhlmann, Th. Roatsch, R. Lundin, C.T. Russel and J.G. LuHMANN (1992): The Martian magnetic field environment: induced or dominated by an intrinsic magnetic field?, $A d v$. Space Res., 12 (9), 213-219. 
SiEgFRed, R.W. and S.C. Solomon (1974): Mercury: internal structure and thermal evolution, Icarus, 23, 192-205.

Soward, A.M. (1992): Dynamo Theory, Adv. Space Res., 12 (8), 257-263.

Stevenson, D.J. (1983): Planetary magnetic fields, Rep. Prog. Phys., 46, 555-620.

Stevenson, D.J. (1984): The energy flux number and three types of planetary dynamos, Astron. Nachr., 305, 257-264.

UCHUPY, E. and K.O. EMERY (1993): Morphology of the rocky members of the Solar System (SpringerVerlag, Berlin), 394.

Voorhies, C. V. and E.R. Benton (1982): Pole- strength of the Earth from Magsat and magnetic determination of the core radius, Geophys. Res. Lett., 9, 258-261.

Yeroshenko, Ye. (1992): The Martian magnetic field: search and perspectives, Adv. Space Res., 12 (8), 219-226.

ZHANG, K.K. and F.H. Busse (1990): Generation of magnetic fields by convection in a rotating spherical fluid shell of infinite Prandtl Number, Phys. Earth Planet. Inter., 5, 208.

(received January 27, 1993; accepted March 9, 1994) 